\title{
THE REFLECTION OF AN ELECTROMAGNETIC PLANE WAVE BY AN INFINITE SET OF PLATES III*
}

\author{
BY \\ ALBERT E. HEINS \\ Carnegie Institute of Technology
}

1. Introduction. In 1947, J. F. Carlson and the present writer [1], [2] investigated the reflection properties of an electromagnetic plane wave by an infinite set of parallel plates. The problem was investigated for two polarizations, that is, the cases for which (a) the electric vector was parallel to the edges of the parallel plates and (b) the magnetic vector was parallel to the edges of the plates. Let us remark in passing, that case (b) is equivalent to a problem in acoustics. One may see this very easily by referring to $\mathrm{CH}$ II and noting that the problem solved there is a scalar electromagnetic problem and one can pair electromagnetic terminology with acoustic terminology and note the equivalence.

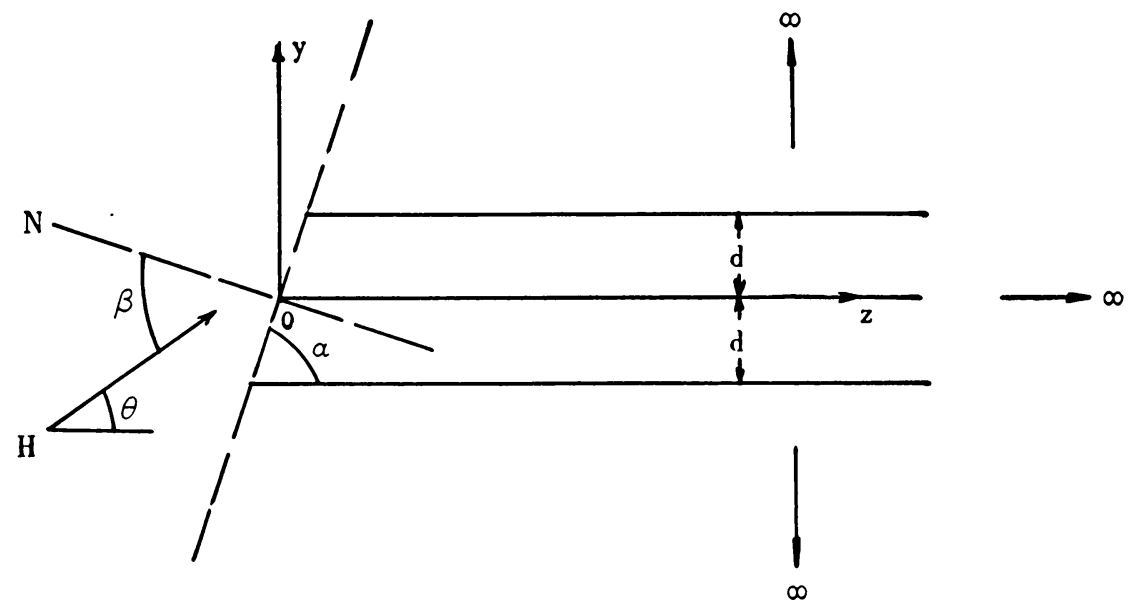

FIG. 1

In $\mathrm{CH}$ I and $\mathrm{CH}$ II, we solved the problems subject to the fact that there be a single propagating mode in the ducts and a single reflected wave in free space. We shall still retain the first hypothesis but shall reinvestigate the second one. We shall be concerned with the case in which there are two reflected waves and shall find the restrictions on wave length, angle of stagger and angle of incidence. We shall also find the new transmission coefficient as well as the two reflection coefficients. As a check, it will be shown that there exists a relation between the magnitudes of these coefficients which can be calculated with the aid of the complex form of the Poynting vector theorem. The case that we consider here is the "E plane" case, the one which has the acoustical analogue.

Since the formulation of this problem has been discussed in $\mathrm{CH}$ I and $\mathrm{CH}$ II, we only summarize the pertinent equations we used in $\mathrm{CH}$ II. We refer to Figure 1 for a view of the structure in any plane parallel to the $y z$ plane.

${ }^{*}$ Received Oct. 19, 1949. The material treated here is taken from an invited paper presented to the U.R.S.I.-I.R.E. meeting in Washington, D. C., on October 31, 1949. 
The angle of incidence of the plane wave is $\theta$; the angle of stagger of the parallel plates, which are semi-infinite in the $z$ direction and infinite in the $x$ direction, is $\alpha$. The line $O N$ is perpendicular to the trace of the edges and the angle $\beta$ [3] is the angle which the direction of propagation of the plane wave makes with this normal. Furthermore we have that $\alpha-\pi<\theta<\alpha$ and $0<\alpha \leq \pi / 2$. It is assumed here that the incident wave has only one component of the magnetic field, $H_{x}(y, z)$, that is, the one which is parallel to the $x$ axis. For such an excitation, no other components of the magnetic field can be excited and hence the electric field may be derived from $H_{x}(y, z)$. If we now assume that the time variation of the field quantities is monochromatic, that is of the form $\exp (-i k c t)$, then the Maxwell equations assume the form

$$
\nabla \times \mathbf{E}=i k \mathbf{H}
$$

and

$$
\nabla \times \mathbf{H}=-i k \mathbf{E}
$$

where $k=2 \pi / \lambda$ and $\lambda$ is the free space wave-length. Here $c$ is the velocity of light in units appropriate to those of $\lambda$. Let us, as a matter of notation, introduce $H_{x}(y, z)=$ $\psi(y, z)$. Then (1.1) and (1.2) reduce to

$$
\frac{\partial^{2} \psi}{\partial y^{2}}+\frac{\partial^{2} \psi}{\partial z^{2}}+k^{2} \psi=0
$$

and this is to be solved subject to the boundary condition that the $z$ component, of the electric field vanishes on $y=n d, z \geq n d \cot \alpha$. If this boundary condition is expressed in terms of $\psi(y, z)$ we see that this is equivalent to the vanishing of $\partial \psi / \partial y$ on $y=n d$, $z \geq n d \cot \alpha, n=0, \pm 1, \cdots$. There are also conditions at infinity which have been described in $\mathrm{CH} \mathrm{I}$.

We recall from $\mathrm{CH}$ II, Eq. (3.2) that this boundary value problem may be formulated as a Wiener-Hopf integral equation for the current density on the plates. The plates are assumed to be perfect conductors of zero thickness. That is

$0=i k \sin \theta \exp [i k z \cos \theta]$

$$
+\frac{i}{4} \sum_{p=-\infty}^{\infty} \int_{0}^{\infty} I_{0}\left(z^{\prime}\right) \exp [i k \mu p] \frac{\partial^{2}}{\partial y^{2}} H_{0}^{(1)}\left[k\left\{\left(z-z^{\prime}+p g\right)^{2}+(y-n d-p d)^{2}\right\}^{1 / 2}\right] d z^{\prime}
$$

where $z \geq 0$. Here $\mu=g \cos \theta+d \sin \theta$, and $g=d \cot \alpha$, while the variable $y$ is set equal to $n d$. Further the surface current density on the $m$ plate is proportional to $I_{m}(z)$ and may be expressed in terms of a single current density by the relation

$$
I_{m}(z-m g)=I_{0}(z) \exp [i k m \mu] .
$$

For all $y$ and $z$ we have then the following Fourier integral representation for $\psi(y, z)$.

$$
\begin{aligned}
\psi(y, z) & =\exp [i k(y \sin \theta+z \cos \theta)]-\frac{1}{4 \pi} \int_{C} d w J(w) \exp [i(w z+k \mu n-w g n)] \\
& \times \frac{\cos \left\{(y-n d-d)\left(k^{2}-w^{2}\right)^{1 / 2}\right\}-\exp [i(k \mu-w g)] \cos \left\{(d n-y)\left(k^{2}-w^{2}\right)^{1 / 2}\right\}}{\cos \left[d\left(k^{2}-w^{2}\right)^{1 / 2}\right]-\cos (k \mu-w g)}
\end{aligned}
$$


where $J(w)$ is

$$
J(w)=\int_{0}^{\infty} I_{0}\left(z^{\prime}\right) \exp \left(-i w z^{\prime}\right) d z^{\prime} .
$$

The path $C$ is drawn within a certain strip of the complex $w$ plane which we shall define in the following section. Here $n$ is the largest integer in the ratio $y / d$. We assume here that $k$ has a small positive imaginary part, and the precise meaning of small will be defined in Sec. 2.

2. The Fourier transform of the kernel of equation (1.4). The Fourier Transform of the kernel of the integral equation (1.4) is

$$
-\frac{i}{2}\left\{k^{2}-w^{2}\right\}^{1 / 2} \sum_{p=-\infty}^{\infty} \exp \left[i p(k \mu-w g)+|p| d\left\{k^{2}-w^{2}\right\}^{1 / 2}\right] .
$$

We choose here that branch of $\left(k^{2}-w^{2}\right)^{1 / 2}$ which reduces to $k$ when $w=0$. The series in (2.1) is not in a particularly useful form since it does not exhibit the singularities of the function it represents, save for the possible branch points at $w= \pm k$. We say possible branch points advisedly, since in closing this sum, we shall find that they disappear and the only singularities possessed by (2.1) are two infinite sequences of poles. In any event, each term of the series has branch points at $w= \pm k$, so that each is regular in the strip $-\Im \mathfrak{m} k<\Im \mathfrak{m} w<\Im \mathfrak{m} k$. We observe further that the series (2.1) diverges when

$$
k \mu-w g+d\left\{k^{2}-w^{2}\right\}^{1 / 2}=2 n \pi
$$

or

$$
k \mu-w g-d\left\{k^{2}-w^{2}\right\}^{1 / 2}=2 n \pi
$$

$n=0, \pm 1, \pm 2, \cdots$. From Eqs. (2.2a) and $(2.2 \mathrm{~b})$ it is clear that the singularities of (2.1) are intimately related to the values of $\alpha, \theta, d$ and $k$ which we use. For $n=0$, we find that $w$ may be $k \cos (2 \alpha-\theta)=\sigma_{2}$ or $k \cos \theta=\sigma_{1}$. Since $\alpha-\pi<\theta<\alpha$ and $0<\alpha \leq \pi / 2$, it is clear that $\Im \mathfrak{m} \sigma_{2}<\Im \mathfrak{m} \sigma_{1}$. From this we see that the series converges at best in a strip $\Im_{\mathfrak{m} \sigma_{2}}<\Im \mathfrak{m} w<\Im \mathfrak{m} \sigma_{1}$ and this is so, provided that there are no other singularities given by Eqs. (2.2a) and (2.2b) which are in a smaller strip.

A next possible root which may be in the strip $\Im_{\mathfrak{m} \sigma_{2}}<\Im \mathfrak{m} w<\Im \mathfrak{m} \sigma_{1}$ comes from Eqs. (2.2a) and (2.2b) by putting $n= \pm 1$. Let us assume that the angle $\beta=\pi / 2+$ $\theta-\alpha$ satisfies the inequality $0<\beta \leq \pi / 2$. Upon solving Eqs. (2.2a) and (2.2b) for $n=1$ we get

$$
w_{1}=(2 \pi / d) \sin \alpha[-\cos \alpha(1-k \mu / 2 \pi)+\Delta]
$$

and

$$
w_{2}=(2 \pi / d) \sin \alpha[-\cos \alpha(1-k \mu / 2 \pi)-\Delta]
$$

where $\Delta$ is given by the expression

$$
\Delta=\left[(d k / 2 \pi)^{2}-\sin ^{2} \alpha(1-k \mu / 2 \pi)^{2}\right]^{1 / 2}
$$

and the positive root is understood for $\Delta$. The roots $w_{1}$ and $w_{2}$ are written in this form under the assumption that $\Delta$ is real for $k$ real. Observe that $w_{1}$ satisfies Eq. (2.2a) while $w_{2}$ satisfies Eq. (2.2b) for $n=1$. 
We now write $\Delta_{r}=\Delta$ for $k$ real and assume further that $\Delta$ does not vanish. Then we may expand $w_{1}$ and $w_{2}$ for $\Im \mathrm{m} k=\epsilon$ sufficiently small (where now $\epsilon$ is small enough to insure the convergence of the expansion) and we obtain

$$
\Im \mathfrak{m} w_{1}=[(\epsilon \sin \alpha) / d]\left[\mu \cos \alpha+\left(d^{2} k_{r}+2 \pi \mu \sin ^{2} \alpha-k_{r} \mu^{2} \sin ^{2} \alpha\right) / 2 \pi \Delta_{r}\right]
$$

and

$$
\Im \mathfrak{m} w_{2}=[(\epsilon \sin \alpha) / d]\left[\dot{\mu} \cos \alpha-\left(d^{2} k_{r}+2 \pi \mu \sin ^{2} \alpha-k_{r} \mu^{2} \sin ^{2} \alpha\right) / 2 \pi \Delta_{r}\right]
$$

if we only retain first powers of $\epsilon$. In terms of the angle $\beta$, these expressions may be simplified to read

$$
\Im_{\mathfrak{m} w_{1}}=\epsilon\left(\cos \alpha \sin \beta+\sin \alpha\left(d k_{r} \cos ^{2} \beta+2 \pi \sin \alpha \sin \beta\right) / 2 \pi \Delta_{r}\right]
$$

and

$$
\Im \mathfrak{m} w_{2}=\epsilon\left[\cos \alpha \sin \beta-\sin \alpha\left(d k_{r} \cos ^{2} \beta+2 \pi \sin \alpha \sin \beta\right) / 2 \pi \Delta_{r}\right] .
$$

Clearly, under the restrictions we have imposed on $\alpha$ and $\beta, \Im \mathfrak{m} w_{2}<\Im \mathfrak{m} w_{1}$ so that it remains to compare the relative orders of magnitude of $\Im \mathfrak{m} w_{1}, \Im \mathfrak{m} w_{2}, \Im \mathfrak{m} \sigma_{1}$ and $\Im \mathfrak{m} \sigma_{2}$. We shall now show that $\Im \mathfrak{m} w_{2}<\Im \mathfrak{m} \sigma_{1}$. This implies that

$$
\cos \alpha \sin \beta-\sin \alpha\left(d k_{r} \cos ^{2} \beta+2 \pi \sin \alpha \sin \beta\right) / 2 \pi \Delta_{r}<\cos \theta
$$

or

$$
\cos \alpha \sin \beta-\sin \alpha\left(d k_{r} \cos ^{2} \beta+2 \pi \sin \alpha \sin \beta\right) / 2 \pi \Delta_{r}<\sin (\alpha+\beta)
$$

From this, we get immediately

$$
-\sin \alpha\left(d k_{r} \cos ^{2} \beta+2 \pi \sin \alpha \sin \beta\right) / 2 \pi \Delta_{r}<\sin \alpha \cos \beta .
$$

Since the left side of this inequality is always negative for $\alpha$ and $\beta$ positive and acute, while the right side is positive, it is clear that $\Im \mathfrak{m} w_{2}<\Im \mathfrak{m} \sigma_{1}$. We have similarly that $\Im \mathfrak{m} \sigma_{2}<\Im \mathfrak{m} w_{1}, \Im \mathfrak{m} \sigma_{1}<\Im \mathfrak{m} w_{1}$, and $\Im \mathfrak{m} w_{2}<\Im \mathfrak{m} \sigma_{2}$. If we now combine all of the inequalities we get

$$
\Im \mathfrak{m} w_{2}<\Im \mathfrak{m} \sigma_{2}<\Im \mathfrak{m} w<\Im \mathfrak{m} \sigma_{1}<\Im \mathfrak{m} w_{1}
$$

Hence the series (2.1) converges in the strip

$$
\Im \mathfrak{m} \sigma_{2}<\Im \mathfrak{m} w<\Im \mathfrak{m} \sigma_{1}
$$

provided now that there are no other roots of Eqs. (2.2a) or (2.2b) in this strip. Such will be the case, if we limit $d k_{r}$ properly. Let us suppose for the present that such is the case.

One observes at this point, that if $\Delta_{r}$ is real for real $k$, that is for $k=k_{r}$, then the following inequality is satisfied by $k_{r}, d, \theta$ and $\alpha$. We have here

$$
\frac{d k_{r}}{2 \pi}>\frac{\sin \alpha}{1+\cos (\theta-\alpha)}
$$

But in order that one mode propagate in the ducts,

$$
0<\left(d k_{r}\right) / 2 \pi<\frac{1}{2}
$$


Hence, subject to the condition that $0<\beta<\pi / 2$, the strip of regularity of $(2.1)$ is as we have given it, provided

$$
0<\frac{\sin \alpha}{1+\cos (\theta-\alpha)}<\frac{d k_{r}}{2 \pi}<\frac{1}{2} .
$$

This inequality on $\left(d k_{r}\right) / 2 \pi$ assures us of the existence of at least the two propagating reflected waves in free space. Since we desire only two such waves, we should put down conditions which insure that the remaining $\Delta_{n}$ for $n=-1, \pm 2, \pm 3, \cdots$ be imaginary for $k_{r}$ real. In the first place, the remaining roots of Eqs. (2.2a) and (2.2b) are

$$
[(2 \pi n \sin \alpha) / d]\left[-\cos \alpha(1-k \mu / 2 \pi n) \pm i \Delta_{n}\right]
$$

where

$$
\Delta_{n}=\left[\sin ^{2} \alpha(1-k \mu / 2 \pi n)^{2}-(d k)^{2} / 4 n^{2} \pi^{2}\right]^{1 / 2} .
$$

$\Delta_{n}$ will vanish if

$$
(d k) / 2 n \pi=-\sin \alpha /[1-\cos (\theta-\alpha)] \quad n= \pm 1, \pm 2, \cdots
$$

or

$$
(d k) / 2 n \pi=\sin \alpha /[1+\cos (\theta-\alpha)] \quad n= \pm 1, \pm 2, \cdots .
$$

But for $n$ negative only, the roots

$$
d k / 2 n \pi=-\sin \alpha /[1-\cos (\theta-\alpha)] \quad n=-1,-2, \cdots
$$

satisfy the inequality $d k / 2 \pi>0$, while for $n$ positive, only the roots

$$
d k / 2 n \pi=\sin \alpha /[1+\cos (\theta-\alpha)] \quad n=1,2, \cdots
$$

satisfy the same inequality.

From the two sequence of roots (2.6) and (2.7) we can now form the condition that there be only two reflected waves. One reflected wave arises from the root $w=\sigma_{2}$. This we have seen in CH II corresponds to the reflected wave which makes an angle with respect to $O N$ equal numerically to that of the incident wave. The other one corresponds to $w=w_{2}$. In order that a third reflected wave not be present

$$
\left(d k_{r}\right) / 2 \pi<(2 \sin \alpha) /[1+\cos (\theta-\alpha)] \quad \text { or } \quad \sin \alpha /[1-\cos (\theta-\alpha)] .
$$

If $d k_{r}$ satisfies this inequality, it satisfies the inequality

$$
\begin{array}{cc}
\left(d k_{r}\right) / 2 \pi<(n \sin \alpha) /[1+\cos (\theta-\alpha)] & 0<\alpha-\theta<\pi / 2 \\
n=3,4, \cdots
\end{array}
$$

as well as

$$
\begin{array}{cc}
\left(d k_{r}\right) / 2 \pi<(n \sin \alpha) /[1-\cos (\theta-\alpha)] & 0<\alpha-\theta<\pi / 2 \\
n=2,3, \cdots .
\end{array}
$$

We have then the following condition for two reflected waves.

$$
\begin{aligned}
0< & (\sin \alpha) /[1+\cos (\theta-\alpha)]<\left(d k_{r}\right) / 2 \pi<\left\{\frac{1}{2} \text { or }(2 \sin \alpha) /[1+\cos (\theta-\alpha)]\right. \\
& \quad \text { or } \sin \alpha /[1-\cos (\theta-\alpha)]\}
\end{aligned}
$$


The term on the right side is to be interpreted as the smaller of the three terms. For example if $\sin \beta=.25$, this inequality reads

$$
0<(4 \sin \alpha) / 5<\left(d k_{r}\right) / 2 \pi<\left\{\frac{1}{2} \text { or }(4 \sin \alpha) / 3\right\}
$$

On the other hand, if $\sin \beta=.5$, this inequality reads

$$
0<(2 \sin \alpha) / 3<\left(d k_{r}\right) / 2 \pi<\left\{\frac{1}{2} \text { or }(4 \sin \alpha) / 3\right\} .
$$

It is clear that we can find conditions for three or more reflected waves by arguments similar to those that we have just used, but we shall not pursue this detail any further. One final remark is in order. Suppose that $\pi / 2<\alpha-\theta<\pi$. In this case the inequality reads

$$
\begin{aligned}
0< & (\sin \alpha) /[1-\cos (\theta-\alpha)]<\left(d k_{r}\right) / 2 \pi<\left\{\frac{1}{2} \text { or }(2 \sin \alpha) /[1-\cos (\theta-\alpha)]\right. \\
& \quad \text { or } \sin \alpha /[1+\cos (\theta-\alpha)]\}
\end{aligned}
$$

with the same interpretation lent to the right side of the inequality as we did for the case $0<\alpha-\theta<\pi / 2$.

3. The solution of equation (1.4). We define Eq. (1.4) for all $z$ to read

$$
\begin{aligned}
F(z)= & \psi_{0}(z) \\
& +\frac{i}{4} \sum_{p=-\infty}^{\infty} \int_{0}^{\infty} I_{0}\left(z^{\prime}\right) \exp [i k \mu p] \frac{\partial^{2}}{\partial y^{2}} H_{0}^{(1)}\left[k\left\{\left(z-z^{\prime}+p g\right)^{2}+(y-n d-d)^{2}\right\}^{1 / 2}\right] d z^{\prime}
\end{aligned}
$$

where $F(z)$ vanishes identically for positive $z$. Further $\psi_{0}(z) \equiv i k \sin \theta \exp [i k z \cos \theta]$ for positive $z$ and vanishes identically for negative $z$. In view of the restrictions we have placed on $d k_{r}, \theta$ and $\alpha$, we have that

$$
F(z)=O\left[\exp \left(i \sigma_{2} z\right)\right]+O\left[\exp \left(i w_{2} z\right)\right]
$$

for $z \rightarrow-\infty$. In order to see this, we rewrite Eq. (3.1) in Fourier integral representation as

$$
\begin{aligned}
F(z)= & \psi_{0}(z)-\frac{1}{4 \pi} \int_{C} d w J(w) \exp [i(w z+k \mu n-w g n)] \\
& \cdot \frac{\left\{k^{2}-w^{2}\right\}^{1 / 2} \sin \left[d\left\{k^{2}-w^{2}\right\}^{1 / 2}\right]}{\cos \left[d\left\{k^{2}-w^{2}\right\}^{1 / 2}\right]-\cos (k \mu-w g)} .
\end{aligned}
$$

Since $I_{0}(z)$ has the same $z$ dependence as the magnetic field, and since only the lowest mode propagates in the ducts, $J(w)$ is regular in the lower half plane $\Im \mathfrak{m} w<\Im \mathfrak{m} k$. Further since there are two reflected waves, the path $C$ is drawn in the strip $\Im \mathfrak{m} \sigma_{2}<$ $\Im \mathfrak{m} w<\Im_{\mathfrak{m} \sigma_{1}}[4]$. In order to find $F(z)$ for $z \rightarrow-\infty$, we need only calculate the residues of the poles in the lower half plane $\Im \mathfrak{m} w<\Im \mathfrak{m} \sigma_{1}$. Upon doing so, we find that the dominant terms are of the form given in Eq. (3.2); all other terms which have been omitted are small compared to these two. This incidently tells us that the unilateral Fourier transform of $F(z)$ is regular in the upper half plane $\Im \mathfrak{m} w>\Im \mathfrak{m} \sigma_{2}$, provided we assume the integrability of $F(z)$ for $z$ negative and finite. This may be verified with the solution of the integral equation (3.1). The Fourier transform of $\psi_{0}(z)$ is regular in the lower 
half plane $\mathfrak{I} \mathfrak{m} w<\mathfrak{I} \sigma_{1}$. Finally the transform of the kernel (2.1) may be written in closed form as

$$
\frac{\left\{k^{2}-w^{2}\right\}^{1 / 2} \sin \left[d\left\{k^{2}-w^{2}\right\}^{1 / 2}\right]}{\cos \left[d\left\{k^{2}-w^{2}\right\}^{1 / 2}\right]-\cos [k \mu-w g]}=\frac{K_{-}(w)}{K_{+}(w)}
$$

and this is regular in the strip $\Im \mathfrak{m} \sigma_{2}<\Im \mathfrak{m} w<\Im \mathfrak{m} \sigma_{1}$. There is then a common strip of analyticity to all transforms involved and it is thus permissable to apply the Fourier transform to Eq. (3.1) to get

$$
D(w)=\frac{k \sin \theta}{w-\sigma_{1}}-\frac{K_{-}(w) J(w)}{2 K_{+}(w)} .
$$

If we separate terms as we did in CH II we get

and

$$
J(w)=\frac{2 k \sin \theta K_{+}\left(\sigma_{1}\right)}{\left(w-\sigma_{1}\right) K_{-}(w)}
$$

$$
D(w)=\frac{k \sin \theta\left[K_{+}(w)-K_{+}\left(\sigma_{1}\right)\right]}{\left(w-\sigma_{1}\right) K_{+}(w)}
$$

The integral function of separation vanishes identically in this case. The terms $K_{-}(w)$ and $K_{+}(w)$ are now given by the following expressions.

$$
K_{-}(w)=N_{-}(w) / D_{-}(w) \quad \text { and } \quad K_{+}(w)=D_{+}(w) / N_{+}(w)
$$

where

$$
\begin{aligned}
N_{-}(w)= & \left\{\exp [x(w)\}\{k-w\} \prod_{n=1}^{\infty}\left[\left\{1-(k d / n \pi)^{2}\right\}^{1 / 2}+i w d / n \pi\right] \exp [-i w d / n \pi]\right. \\
N_{+}(w)= & \{2 d(k+w) \\
& \left.\quad \exp [-\chi(w)] /\left(d^{2}+g^{2}\right)\right\} \prod_{n=1}^{\infty}\left[\left\{1-(k d / n \pi)^{2}\right\}^{1 / 2}-i w d / n \pi\right] \exp [i w d / n \pi] \\
D_{-}(w) & =\left(w-\sigma_{1}\right)\left\{\prod_{n=-\infty}^{-1}\left[\Delta_{n}-i \Psi_{n}\right] \exp [(k \mu-w g+i w d) / 2 n \pi+i(\pi / 2-\alpha)]\right\} \\
& \times\left\{\prod_{n=2}^{\infty}\left[\Delta_{n}+i \Psi_{n}\right] \exp [(k \mu-w g-i w d) / 2 n \pi-i(\pi / 2-\alpha)]\right\} \\
& \times\left\{\left(\Psi_{1}-\Delta\right) \exp [(k \mu-w g+i w d) / 2 \pi-i(\pi / 2-\alpha)]\right\}
\end{aligned}
$$

and

$$
\begin{aligned}
D_{+}(w) & =\left(w-\sigma_{2}\right)\left\{\prod_{-\infty}^{-1}\left[\Delta_{n}+i \Psi_{n}\right] \exp [(k \mu-w g-i w d) / 2 n \pi-i(\pi / 2-\alpha)]\right\} \\
& \times\left\{\prod_{n=2}^{\infty}\left[\Delta_{n}-i \Psi_{n}\right] \exp [(k \mu-w g+i w d) / 2 n \pi+i(\pi / 2-\alpha)]\right\} \\
& \times\left\{\left(\Psi_{1}+\Delta\right) \exp [(k \mu-w g-i w d) / 2 \pi+i(\pi / 2-\alpha)]\right\}
\end{aligned}
$$


Here

$$
\begin{aligned}
& \Delta_{n}=\left\{\sin ^{2} \alpha(1-k \mu / 2 n \pi)^{2}-(k d)^{2} / 4 n^{2} \pi^{2}\right\}^{1 / 2} \quad n=-1, \pm 2, \cdots \\
& \Delta=\left\{(k d)^{2} / 4 \pi^{2}-\sin ^{2} \alpha(1-k \mu / 2 \pi)^{2}\right\}^{1 / 2} \\
& \Psi_{n}=[\cos \alpha(1-k \mu / 2 n \pi)]+(w d \csc \alpha) / 2 n \pi \quad n= \pm 1, \pm 2, \cdots
\end{aligned}
$$

and

$$
\chi(w)=-[i w d / 2 \pi][(\alpha-\pi / 2) \cot \alpha-\ln (2 \sin \alpha)] .
$$

The subscripts - and + on $K, N$ or $D$ indicate that the function is regular [5] in the lower or upper half plane, as the case may be. For example $D_{-}(w)$ is regular in the lower half plane $\Im \mathfrak{m} w<\Im \mathfrak{m} \sigma_{1}, N_{-}(w)$ is regular in the lower half plane $\Im \mathfrak{m} w<\Im \mathfrak{m} k$, etc.

4. The investigation of the far fields. We have found $J(w)$, the Fourier transform of $I_{0}(z)$. If we now use Eq. (1.5), we have an integral representation for $\psi(y, z)$. From this representation we can determine $\psi(y, z)$ for $z \rightarrow \infty$ (in the ducts) and $\psi(y, z)$ for $z \rightarrow-\infty$, (in free space). For $z>0$, we close the path $C$ in the upper plane [6] and obtain

$$
\begin{gathered}
\psi(y, z)=\frac{i \sin \theta K_{+}\left(\sigma_{1}\right)}{2 d\left(k-\sigma_{1}\right) K_{+}(k)}[1-\exp (i k \mu-i k g)] \exp [i(k z+k \mu n-k g n)] \\
+O\left[(\cos \pi y / d) \exp \left[-z\left\{\pi^{2} / d^{2}-k^{2}\right\}^{1 / 2}\right]\right]
\end{gathered}
$$

Hence for $z \rightarrow \infty, \psi(y, z)$ is asymptotic to

$$
\frac{i \sin \theta K_{+}\left(\sigma_{1}\right)}{2 d\left(k-\sigma_{1}\right) K_{+}(k)}[1-\exp (i k \mu-i k g)] \exp [i(k z+k \mu n-k g n)]
$$

and from this we can find the transmission coefficient in the duct. It is

$$
T=\frac{i \sin \theta K_{+}\left(\sigma_{1}\right)}{2 d\left(k-\sigma_{1}\right) K_{+}(k)}[1-\exp (i k \mu-i k g)] .
$$

For $k$ real, $|T|^{2}$ reduces to

$$
|T|^{2}=\left(\frac{\Psi_{1}-\Delta}{\Psi_{1}+\Delta}\right)_{k}\left(\frac{\Psi_{1}+\Delta}{\Psi_{1}-\Delta}\right)_{\sigma_{1}} \frac{\sin ^{2}(\alpha-\theta)}{\left[\cos ^{2} \theta / 2\right]\left[\sin ^{2}(\alpha-\theta / 2)\right]}
$$

where the subscripts $k$ and $\sigma_{1}$ on the first two fractions indicate that $w$ has been evaluated at these values.

For $z<0$, we close the path $C$ in the lower half plane and obtain

$$
\begin{aligned}
\psi(y, z)=\exp [i k(y \sin \theta+z \cos \theta)] \\
+\frac{J\left(\sigma_{2}\right) \exp [i k\{y \sin (2 \alpha-\theta)+z \cos (2 \alpha-\theta)\}]}{2 d[\cot (2 \alpha-\theta)-\cot \alpha]} \\
-\frac{J\left(w_{2}\right)\left\{k^{2}-w_{2}^{2}\right\}^{1 / 2} \exp \left[i\left\{w_{2} z+\left(k^{2}-w_{2}^{2}\right)^{1 / 2}\right\}\right]}{4 \pi(\csc \alpha) \Delta}+
\end{aligned}
$$


terms which attenuate exponentially for $z \rightarrow-\infty$. The term $\left(k^{2}-w_{2}^{2}\right)^{1 / 2}$ may be written as $[2 \pi / d]\left[\Delta \cos \alpha-\sin ^{2} \alpha(1-k \mu / 2 \pi)\right]$. Hence the first reflection coefficient is

$$
R_{1}=J\left(\sigma_{2}\right) / 2 d[\cot (2 \alpha-\theta)-\cot \alpha]
$$

while the second reflection coefficient is

$$
R_{2}=-J\left(w_{2}\right)\left(k^{2}-w_{2}^{2}\right)^{1 / 2} / 4 \pi \Delta \csc \alpha .
$$

For $k$ real

$$
\left|R_{1}\right|^{2}=\frac{\sin ^{2} \theta}{\left(\sigma_{2}-\sigma_{1}\right)^{2} \sin ^{2}(2 \alpha-\theta)}\left|\frac{K_{+}\left(\sigma_{1}\right)}{K_{+}^{\prime}\left(\sigma_{2}\right)}\right|^{2}
$$

where the prime in $K_{+}(w)$ denotes differentiation with respect to the argument $w$. Upon simplifying further, we get finally for $k$ real

$$
\left|R_{1}\right|^{2}=\left[\cot ^{2}(\alpha-\theta / 2)\right]\left[\tan ^{2} \theta / 2\right]\left[\frac{\Psi_{1}-\Delta}{\Psi_{1}+\Delta \Delta}\right]_{\sigma_{2}}\left[\frac{\Psi_{1}+\Delta}{\Psi_{1}-\Delta}\right]_{\sigma_{1}} .
$$

Furthermore for $k$ real

$$
\left|R_{2}\right|^{2}=\frac{4 k^{2}\left[\sin ^{2} \alpha\right]\left[\tan ^{2} \theta / 2\right]\left[\sin ^{2}(\alpha-\theta)\right]}{\left[w_{2}-\sigma_{1}\right]\left[w_{2}-\sigma_{2}\right]}\left[\frac{\Psi_{1}+\Delta}{\Psi_{1}-\Delta}\right]_{\sigma_{1}}\left[\frac{k+w_{2}}{k-w_{2}}\right] .
$$

The values which we have given for $|T|^{2},\left|R_{1}\right|^{2}$ and $\left|R_{2}\right|^{2}$ have been derived subject to the conditions

$$
0<\frac{\sin \alpha}{1+\sin \beta}<\frac{d k}{2 \pi}<\left\{\frac{1}{2} \text { or } \frac{2 \sin \alpha}{1+\sin \beta} \text { or } \frac{\sin \alpha}{1-\sin \beta}\right\}
$$

$$
0<\beta<\pi / 2 \text {. }
$$

The modification for $-\pi / 2<\beta<0$ is quite direct. We observe that for this case, $\pi / 2<\alpha-\theta<\pi$. The requirement for two reflected waves for this interval of $\alpha-\theta$ is

$$
0<\frac{\sin \alpha}{1-\sin \beta}<\frac{k d}{2 \pi}<\left\{\frac{1}{2} \text { or } \frac{2 \sin \alpha}{1-\sin \beta} \text { or } \frac{\sin \alpha}{1+\sin \beta}\right\} \text {. }
$$

In view of the requirement on $\alpha-\theta$, it is clear that the inequalities (a) and (c) are equivalent. There are a number of modifications in the expressions for $\left|R_{1}\right|^{2},\left|R_{2}\right|^{2}$, and $|T|^{2}$ for the range $-\pi / 2<\beta<0$, but we shall not pursue the matter further.

5. Conservation of power flow. As a check for our calculations of $|T|,\left|R_{1}\right|$ and $\left|R_{2}\right|$ we shall now see what information we can derive from the Poynting vector theorem. In the first place, the power flow from free space into any duct is (per unit length in the $x$ direction)

$$
\Re \mathrm{e} \int_{0}^{d}\left(\frac{\csc \alpha}{2}\right)\left(\mathbf{E} \times \mathbf{H}^{*}\right) \cdot \mathbf{n} d y
$$

where $\mathbf{n}$ is the normal vector into the parallel plate region. In terms of $\psi$, this may be written as

$$
\Re e \frac{i \csc \alpha}{2} \int_{0}^{d}\left[\cos \alpha \frac{\partial \psi}{\partial y}-\sin \alpha \frac{\partial \psi}{\partial z}\right] \psi^{*} d y
$$


Here $y=z \tan \alpha$, that is we are integrating over the opening of a parallel plate region. Hence upon simplifying the parenthesis and integrating over $y$, we get

$$
\frac{d \csc \alpha}{2}\left[k \sin (\alpha-\theta)-k\left|R_{1}\right|^{2} \sin (\alpha-\theta)-2 \pi \Delta\left|R_{2}\right|^{2} / d\right] .
$$

This represents the average flow of power into a given duct per unit length in the $x$ direction. This in turn is to be balanced by the average flow of power far down the duct, that is as $z \rightarrow \infty$, nd $<y<(n+1) d$. The magnetic field in the duct for $z \rightarrow \infty$, nd $<$ $y<(n+1) d$ is

$$
\psi\left(y_{1} z\right)=T_{n} \exp [i k z] .
$$

Hence the power flow down any duct for $z \rightarrow \infty$ is $|T|^{2} d / 2$ (recall that $\left|T_{n}\right|$ is independent of $n$, the duct number). Hence conservation of power flow demands that

$$
k|T|^{2}=\csc \alpha\left[k \sin (\alpha-\theta)-k\left|R_{1}\right|^{2} \sin (\alpha-\theta)-2 \pi \Delta\left|R_{2}\right|^{2} / d\right] .
$$

Upon substituting in $\left|R_{1}\right|^{2}$ and $\left|R_{2}\right|^{2}$ we find that Eq. (4.2) is an identity, thus verifying the conservation theorem.

6. Some concluding remarks. If we try to find conditions for higher reflected waves, that is the cases corresponding to $n=-1,-2, \cdots$ or $n=2,3, \cdots$, we find that the conditions for a given number of reflected waves become fairly complex. On the other hand, a study of the zero of the $\Delta_{n}$ 's and their ordering will show us how each reflected wave enters. These results will depend on $\alpha$ and $\beta$. The case $\beta=0$, has been excluded from our present discussion, but it offers no difficulties. In this case, we get higher reflected waves in pairs. The result is not surprising since the incident wave normal and the normal to the trace of the edges of the plates are parallel.

For the case of a single reflected wave, we noted that the reflected wave has a normal whose direction is independent of $k$. In the case of two reflected waves, the first reflected wave has a normal whose direction is independent of $k$, but the second reflected wave has a direction which is dependent on $k$. We shall present a discussion of the numerical results elsewhere.

Finally, we observe that $\left|R_{1}\right|^{2},\left|R_{2}\right|^{2}$ and $|T|^{2}$ have not been normalized. If we refer back to Eq. (4.2) and divide through by $k \csc \alpha \sin (\alpha-\theta)$, the normalized first and second reflection coefficients, as well as transmission coefficients for the case discussed, are respectively

$$
\left|R_{1}\right|^{2}, \quad 2 \pi \Delta \csc (\alpha-\theta)\left|R_{2}\right|^{2} / d \quad \text { and } \quad|T|^{2} \sin \alpha \csc (\alpha-\theta) .
$$

For the normalized coefficients, all magnitudes are numerically less than unity.

\section{References aNd Notes.}

1. J. F. Carlson and A. E. Heins The reflection of an electromagnetic plane wave by an infinite set of plates, $I$, this Quarterly, 4, 313-329 (1947). Hereafter we shall refer to this paper as CH I.

2. A. E. Heins and J. F. Carlson The reflection of an electromagnetic plane wave by an infinite set of plates, $I I$, this Quarterly, 5, 82-88 (1947). Hereafter we shall refer to this paper as CH II.

3. If the propagation normal of the incident wave falls to the left of $O N$, angle $\beta$ is a positive acute angle, while if it falls to the right of $O N$, the angle $\beta$ is a negative acute angle. The case $\beta=0$ requires separate treatment. 
4. At this point we realize that to get more than two reflected waves, the inequalities in sec. 2 have to be modified. As we carry on this modification we find that an indefinite number of reflected waves cannot enter. For example, if $\alpha=\pi / 2$, then the inequality for two reflected waves reads

$$
0<\frac{1}{1+\sin \theta}<\frac{d k}{2 \pi}<\left\{\frac{1}{2} \text { or } \frac{2}{1+\sin \theta} \text { or } \frac{1}{1-\sin \theta}\right\}
$$

for $0<\theta<\pi / 2$. This is impossible since $\sin \theta<1$. Hence there is only one reflected wave in this case. The formulation we gave in CH I makes no assumptions as to the form of $\psi(y, z)$ to the left of the parallel plates. The convergence study in sec 2 , of the present paper gives us conditions for one, two, etc., reflected waves.

5. See CH I, p. 321 for some remarks regarding the present use of the term regular.

6. See CH I, sec. 4. 